\title{
Cognitive suppression of appetite less efficient in women than in men
}

Women might be less efficient than men are at suppressing their desire to eat. In a study of 23 healthy individuals, cognitive inhibition decreased brain responses to food stimuli in men but not in women. "We demonstrated for the first time that women don't have the same ability to inhibit their desire to eat when exposed to their favorite foods," summarized Gene-Jack Wang, Senior Medical Scientist at Brookhaven National Laboratory, Upton, New York, NY.

\section{After cognitive inhibition ...}

PET scans ... revealed changes in brain activity only in men $\mathbf{7 7}$

Previous studies by the same group identified regional metabolic changes in the brain in response to food stimuli, including increased activity of the orbitofrontal cortex, a region of the brain involved in behavioral control and planning. To explore the neurobiological mechanisms that are involved in the voluntary control of food intake, Wang and colleagues examined the effect of cognitive inhibition on these brain responses.

The investigators used PET scans and fludeoxyglucose to monitor brain activity in 13 women and 10 men with normal body weight; patients fasted for nearly $20 \mathrm{~h}$ before each of three separate scans, which were performed in random order. On one scan day, participants were presented with their favorite foods, which they were free to smell, taste and observe, but not to eat. On another day, they were instructed to suppress their desire for food (for example, by shifting their thoughts) before being tempted with the same foods. A control scan with no food presentation was performed on another day. After cognitive inhibition, both men and women reported reduced hunger and desire for the presented food; PET scans, however, revealed changes in brain activity only in men. These changes were detected in limbic and paralimbic brain areas that are known to be activated by food stimuli.

"These findings provide a new target for development of obesity medication," says Wang, "and may open up a whole new area of research that could lead to better ways to help women control their weight."

\section{Julianna Vig}

Original article Wang, G.-J. et al. Evidence of gender differences in the ability to inhibit brain activation elicited by food stimulation. Proc. Natl Acad. Sci. USA 106, 1249-1254 (2009). 